\title{
ODCR: Energy Efficient and Reliable Density Clustered-based routing protocol for emergency sensor applications
}

\author{
Mohammed S. Al-kahtani, Lutful Karim and Nargis Khan \\ Price Sattam Bin Abdul Aziz University, Al-Kharj, Saudi Arabia and \\ School of ICT, Seneca College of Applied Arts and Technology, Toronto, Canada
}

Protocol for emergency sensor applications

\begin{abstract}
Designing an efficient routing protocol that opportunistically forwards data to the destination node through nearby sensor nodes or devices is significantly important for an effective incidence response and disaster recovery framework. Existing sensor routing protocols are mostly not effective in such disaster recovery applications as the networks are affected (destroyed or overused) in disasters such as earthquake, flood, Tsunami and wildfire. These protocols require a large number of message transmissions to reestablish the clusters and communications that is not energy efficient and result in packet loss. This paper introduces ODCR - an energy efficient and reliable opportunistic density clustered-based routing protocol for such emergency sensor applications. We perform simulation to measure the performance of ODCR protocol in terms of network energy consumptions, throughput and packet loss ratio. Simulation results demonstrate that the ODCR protocol is much better than the existing TEEN, LEACH and LORA protocols in term of these performance metrics.
\end{abstract}

Keywords Mobile sensor network, Opportunistic routing, Density clustering, Disaster routing,

Energy Efficient, Throughput, Packet loss ratio

Paper type Original Article

\section{Introduction}

Among many applications wireless sensor networks had been used for incidence respone and disaster recovery. However, communications among devices are affected in disastrous situations such as earthquake, wildfire, Tsunami as the networks are usually destroyed or inaccessible in disastrous situations and emergency personnel cannot communicate each

(C) Mohammed S. Al-kahtani, Lutful Karim and Nargis Khan. Published in Applied Computing and Informatics. Published by Emerald Publishing Limited. This article is published under the Creative Commons Attribution (CC BY 4.0) license. Anyone may reproduce, distribute, translate and create derivative works of this article (for both commercial and non-commercial purposes), subject to full attribution to the original publication and authors. The full terms of this license may be seen at http:// creativecommons.org/licences/by/4.0/legalcode

Declaration of Competing Interest: The authors declare that they have no known competing financial interests or personal relationships that could have appeared to influence the work reported in this paper.

Publishers note: The publisher wishes to inform readers that the article "ODCR: Energy Efficient and Reliable Density Clustered-based routing protocol for emergency sensor applications" was originally published by the previous publisher of Applied Computing and Informatics and the pagination of this article has been subsequently changed. There has been no change to the content of the article. This change was necessary for the journal to transition from the previous publisher to the new one. The publisher sincerely apologises for any inconvenience caused. To access and cite this article, please use Mohammed S. Al-kahtani, Lutful Karim, Nargis Khan, (2020) "ODCR: Energy Efficient and Reliable Density Clustered-based routing protocol for emergency sensor applications", Applied Computing and Informatics. Vol. ahead-of-print No. ahead-of-print. https://10.1016/j.aci.2020.03.003. The original publication date for this paper was 25/03/2020.
Received 5 October 2019 Revised 22 January 2020 Accepted 22 March 2020 
other through their wireless device to get proper information for quick and proper incident responses. Hence, an alternative solution such as building up an opportunistic network and opportunistic data forwarding could be a better option in these types of emergency scenarios.

Existing solutions construct Mobile Ad hoc Networks [1-4] distributing antennas in the affected area, which are not realistic for disaster recovery. Existing research works [5-8] also suggest wireless opportunistic networks. These networks are constructed using wireless devices, sensors and RFID that are used in everyday use devices and objects e.g., smartphone, tablet, PDA, vehicles, appliances, furniture. The idea of building an opportunistic recovery network for communication using those sensors may save many lives in disastrous situations. Thus, this type of solution provides improvement and efficiency for the emergency rescue teams.

Existence of a network having an end-to-end connection between the sender and receiver is not realistic in a disaster scenario. Traditional data forwarding methods in such scenarios most likely result in a data delivery failure. Hence, a new type of data forwarding and routing protocol with higher throughput and data delivery rate is greatly required for such disastrous situations. Opportunistic forwarding is an approach based on the mobility of nodes in a network where sensors can efficiently forward information to the first responders. In this approach, nodes carry stored messages while moving around and forward them whenever an opportunity arises. This opportunity is simply making a contact with a node that knows how to route the message closer to the desired receiver. Hence, a route is built dynamically from the sender to the receiver. Any node can possibly be the next hop in an opportunistic forwarding. Figure 1 demonstrates such an opportunistic network.

However, designing an energy efficient and reliable opportunistic routing protocol [8,9-17,20-22] poses a great challenge due to the mobility of nodes (i.e., rescue team members) and low transmission ranges of devices (i.e., smartphone, sensors, RFID, Bluetooth). Hence, this paper introduces an energy efficient and reliable opportunistic density clustered-based routing protocol (ODCR) for emergency/disastrous situations. This

Figure 1.

Opportunistic network for disaster recovery.

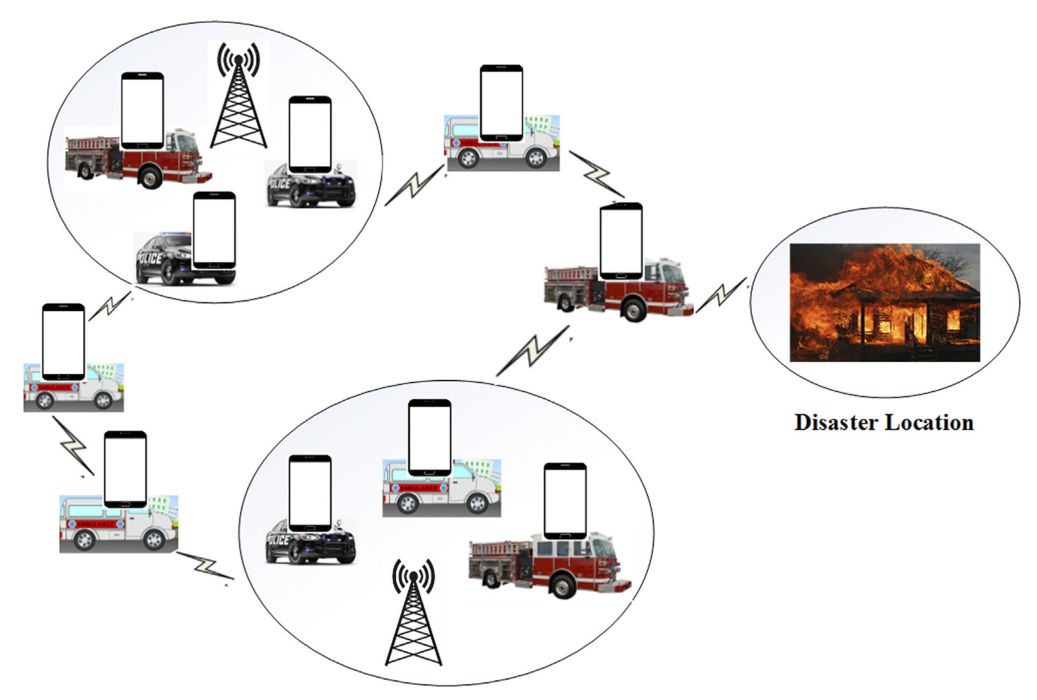


protocol is characterized by an efficient management of the energy and an opportunistic communication aspect to tolerate delays and disruption and build routes in a dynamic and infrastructure-less manner. The number of sensors and quantity of generated data extremely influences the performance of any routing protocol. Hence, the proposed ODCR protocol considers proper data exchange and data aggregation approach and uses a density clustering approach [7,9,10-11].

The proposed ODCR protocol uses opportunistic data forwarding. It uses the concept of both density-based clustering and isolated nodes. In density-based clustering each node is expected to reside in a cluster and exchanges information among the nodes in clusters. On the other hand, isolated nodes are not part of a cluster and forward data packets opportunistically to the node, which has the highest data delivery probability among a number of nodes. Figures 2-4 illustrate such an opportunistic network based on the ODCR protocol where both nodes in cluster and isolated nodes opportunistically create a data transmission path to forward data to the destination. This protocol forms clusters evaluating the Time to Return (TTR) parameter between nodes. Energy consumption of a node varies
Protocol for emergency sensor applications

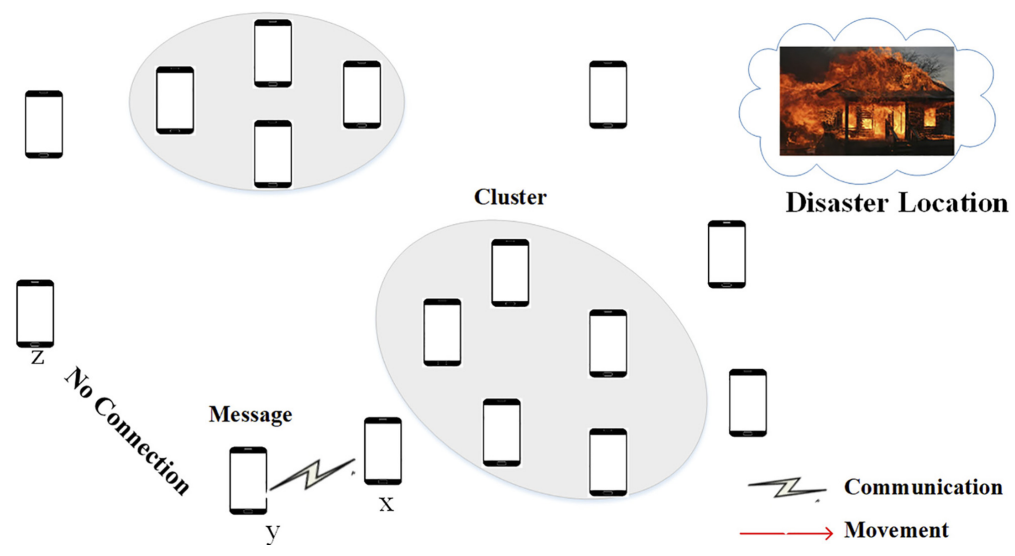

Figure 2.

Disaster network topology.

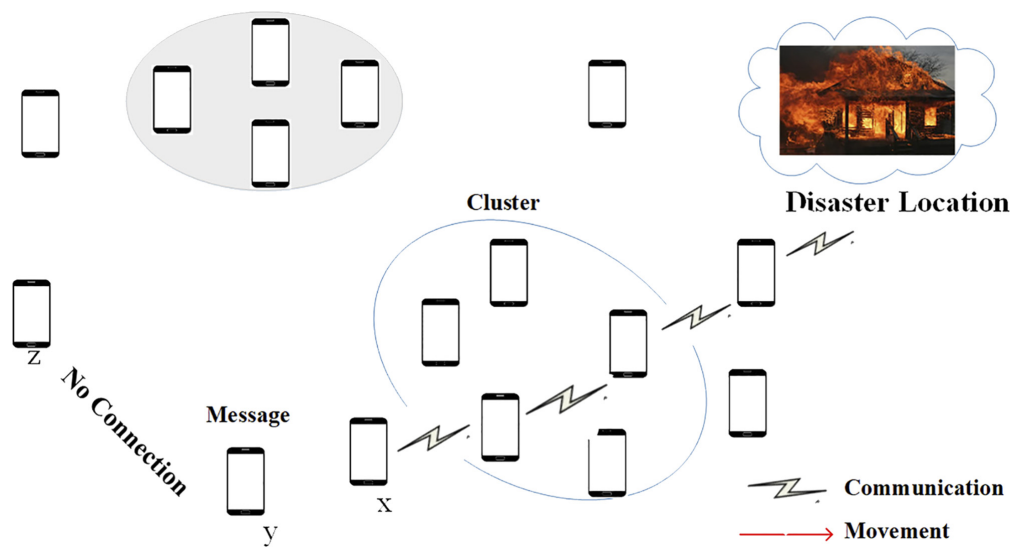

Figure 3. Opportunistic message forwarding in disaster network. 


\section{$\mathrm{ACI}$}

Figure 4.

Cluster-based message transmission.

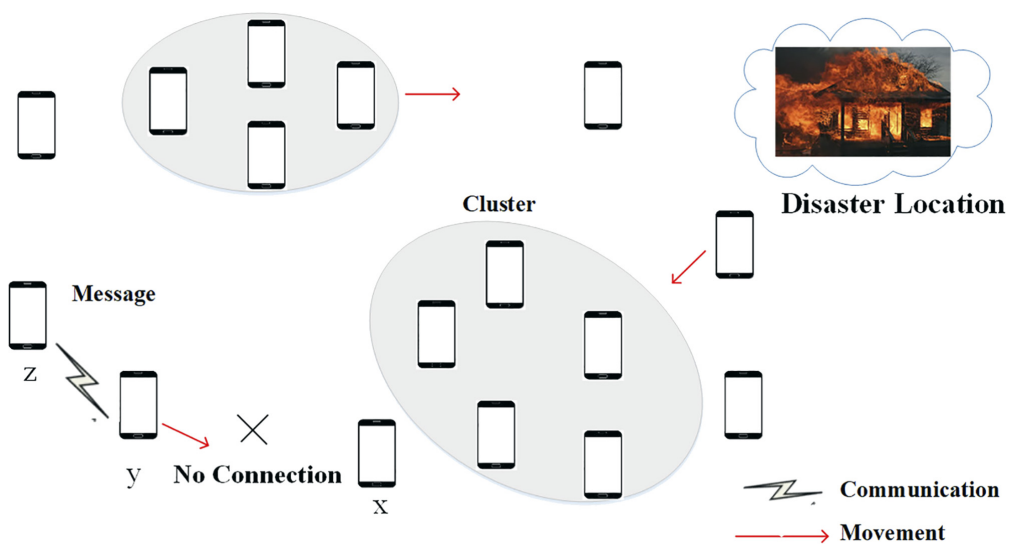

and depends on the role of each node in the cluster. We perform an experiment (simulation) on a realistic disaster area model [8] to measure the performance of the proposed opportunistic routing protocol. Simulation results show that the proposed ODCR protocol in opportunistic forwarding or routing increases the network lifetime and throughput and reduces packet loss ratio as compared to the well-known forwarding protocols such as LEACH, TEEN and LORA $[18,19,21]$.

The rest of the paper is organized as follows. Section 2 presents literature on existing opportunistic routing protocols. Section 3 presents the working principle of the proposed opportunistic density clustered-based routing protocol (ODCR). Section 4 evaluates the performance of the proposed ODCR protocol. Section 5 presents network, energy and mobility models used in the simulation along with simulation setup and results. Finally, Section 6 concludes the paper with some future research direction.

\section{Related work}

Low Energy Adaptive Clustering Protocol (LEACH) [18] is a well- known clustering protocol of sensor network. In this protocol, the cluster head $(\mathrm{CH})$ is randomly selected from all nodes based on a metric called probability of $\mathrm{CH}$. Once clusters are constructed, the member nodes of each cluster transmit data to its $\mathrm{CH}$ using time division multiple access (TDMA) scheme. The $\mathrm{CH}$ collects all data, aggregates and transmits to the base station for further processing. In LEACH, the cluster member nodes transmit data periodically and the cluster formation takes place in every round, which is not energy efficient. In Threshold Sensitive Energy Efficient Sensor Networks Protocol (TEEN) [19] each sensor node senses and transmits an event of interest (e.g., pressure, humidity) based on the minimum attribute value set into to the sensor. The next time the node senses and transmits another event of interest if the attribute value changes by a certain threshold that is set into the node. Hence, the number of sensed and transmitted event is less in TEEN protocol. However, LEACH and TEEN protocols may not work well for emergency and disastrous situation.

The work done in [12] introduced an opportunistic routing protocol, namely EXOR. In this protocol, if the sender needs to transmit data to a destination it first transmits data to a node from its forwarding list, which has the least distance to the actual destination. Once the sender sends the data packet to all forwarding nodes, it determines the best forwarder based on the 
receiving ACK packet from the forwarder nodes. Although EXOR has higher throughput it only uses the information during the time of transmission but does not use the updated information during transmission. Another work in [12] introduces an opportunistic routing that select the next hop forwarding node based on the data delivery ratio and priority of nodes to transmit data.

In [12], the authors present Trust Opportunity Forwarding Mechanism that considers both distance of forwarding node to the destination (cost) and trust factor/degree. The trust factor of a node is calculated by neighboring node through direct observation or recommendations. A node is selected as a forwarder if it has better trust degree as well as less distance to the destination. On the other hand, among several routing protocols Social Relation Opportunistic Routing (SROR) identifies the forwarding nodes based on social relations and profiles among nodes. The work done in [20] introduces a planned opportunistic delay tolerant routing protocol by creating a subset of opportunistic links from source to destination as opposed to deterministic single copy or multiple copies of links between source to destination. This paper optimizes the multiple deterministic links to have only the best one in terms of lower data delivery rate. However, this approach does not employ clustering approach and is not efficient as the creating paths require lot of computations and consumes energy of sensor nodes. The work done in [21] introduces LORA - a load balancing opportunistic routing for asynchronous duty-cycled WSN. To avoid flooding of packets from source node through a number of preselected forwarder nodes this approach creates candidate zones where all possible data forwarder candidate resides. The source node only chooses a candidate based on an OR metric calculated using direction distribution, transmission-distance distribution, perpendicular-distance distribution, and residual energy distribution. Again, this approach seems computationally expensive and does not allow clustering to achieve more energy efficiency. In opportunistic routing, each node decides about the forwarder node locally using greedy approaches and collects lot of information (i.e., overhead) for making such decision. To mitigate this problem, the work done in [22] introduces SeeR - a simulated annealing-based routing protocol for opportunistic mobile networks. This approach calculates a cost function for each node using information such as hop-count and the average aggregated inter-contact time of the node. If the cost of a forwarder node is low the sender transmits the data to that node. In this approach a node does not track information about other nodes, thus, reduces privacy and security risks. However, this approach does not consider clustering. This work done in [23] introduces an opportunistic routing protocol (ORR) that finds the optimal number of forwarders based on a cost function using duty cycle and network topology. Moreover, this approach selects the forwarder from a list of forwarder with more residual energy.

Opportunistic networks use epidemic routing protocols [11] where a node broadcasts or forwards messages to all other nodes that result in high message overhead. To mitigate this problem, the work done in [13] proposes an energy efficient opportunistic routing protocol where a node forwards a packet based on its remaining energy and delivery predictability factor. However, most routing protocols of opportunistic networks do not consider real-time message delivery. The work done by Santos et al. [14] introduces a real-time message propagation model with a maximum data transmission latency for opportunistic networks containing heterogeneous devices.

While existing works mostly consider delay tolerant networks (DTN) to forward messages opportunistically to the destination node they do not consider transmitting messages to the first responders, governmental agency, hospitals and other related organizations to respond to disaster situation. In [15] the authors introduce a routing protocol comprising DTN and Information Centric Network (ICN). The DTN transfers data to the destination and the ICN propagates interest messages to the responding authorities. However, these routing protocols may not work well in emergency or 
disastrous situations. Hence, an efficient opportunistic routing protocol is greatly required for emergency disastrous situation with higher packet deliver ratio and lower latency.

\section{Proposed opportunistic routing protocol}

The proposed ODCR protocol uses the concept of density-based clustering. Moreover, in this protocol, each node forwards or broadcasts its stored data to the nearby or closest node towards the destination as the nodes are mobile and may loss the network connectivity. Section 3.1 presents terminologies used in the proposed ODCR protocol and Sections 3.2-3.4 present different phases of the protocol as follows.

\subsection{Terminologies}

3.1.1 Minimum period of connectivity. In opportunistic networks the minimum period of connectivity (MPC) represents the connectivity strength between two nodes while they are moving. This property also represents successful data packets transmission by a node. The MPC (represented in time) between two nodes $a$ and $b$ is denoted as

$$
M P C_{a b}=\frac{R_{c}-d}{V_{a}+V_{b}}
$$

where $R c$ is the communication range of nodes $a$ and $b, d$ is the distance between $a$ and $\mathrm{b}$. $V_{a}$ is the velocity of node $a$ and $V_{b}$ is the velocity of node $b$.

Global Minimum Period of Connectivity $\left(M P C_{g}\right)$ is a predefined parameter given to the proposed protocol in order to set up a network having clusters with the same $M P C_{g}$ between its nodes.

3.1.2 Weight. The weight of a node represents whether the node is stable or not. The weight of a node is calculated as follows.

$$
\begin{aligned}
Y & =\text { Total number of neighbors of node } a \\
& =\mid \text { neighbors }(a) \mid \\
X & =\sum_{b \in \text { neigbors }(a)} \frac{M P C_{a b}}{M P C_{g}} \\
\text { Weight } & =X-Y
\end{aligned}
$$

\subsection{Density-based cluster formation}

Initially, each node calculates its weight, defined by eq. (3). If the weight of a node $x$ is close to zero the minimum period of connectivity (MPC) of that node to all its neighbors is close to global $M P C\left(M P C_{g}\right)$. That means the node $x$ is more stable and a good candidate to become $\mathrm{CH}$. Once all opportunistic nodes or sensors calculate their weight based on equations (1)(3), they broadcast their weight. The node $x$ with the minimum weight will elect itself as a $\mathrm{CH}$ and broadcasts a "hello" message to let other nodes know that $x$ is a $\mathrm{CH}$. Other nodes will join to a cluster whose $\mathrm{CH}$ is closer to the node. Thus, clusters of the network are formed. Algorithm I presents the pseudocode to select $\mathrm{CH}$, cluster member and border node of a cluster $\mathrm{C}$. 


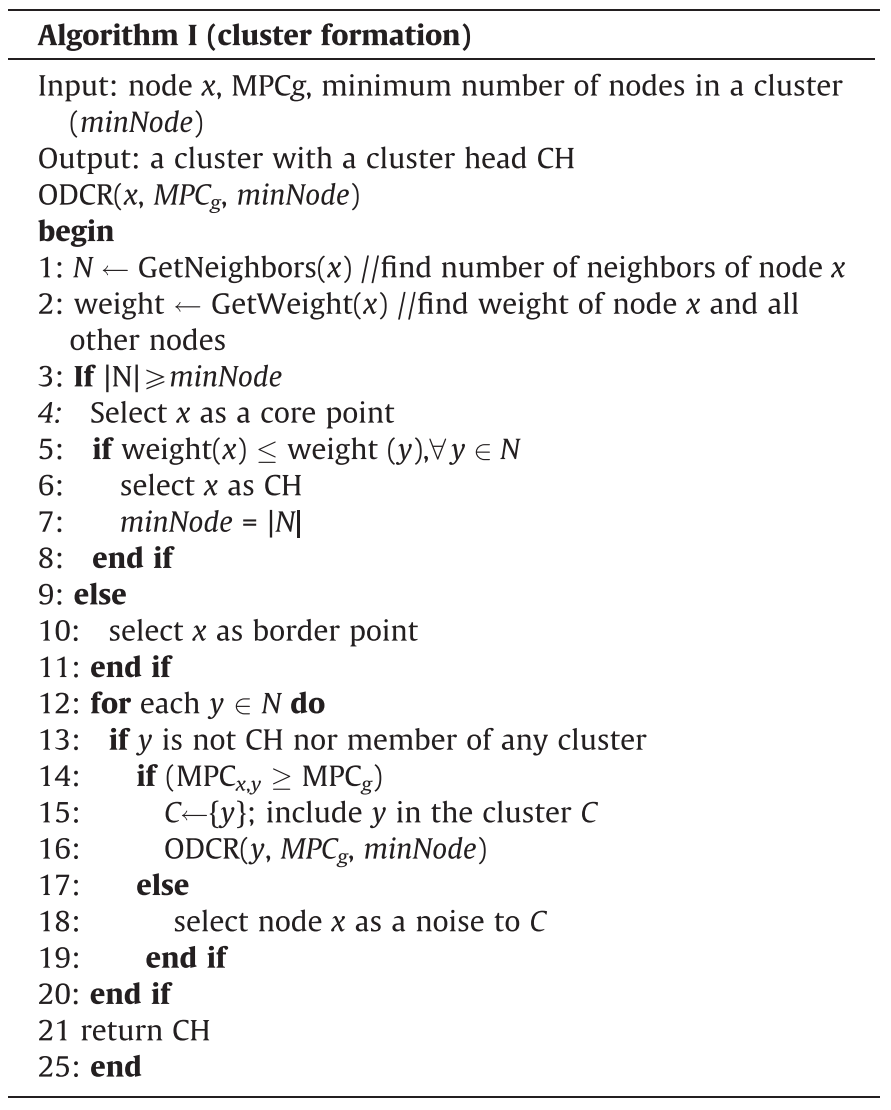

Output: a cluster with a cluster head $\mathrm{CH}$

$\operatorname{ODCR}\left(x, M P C_{g}\right.$, minNode $)$

begin

1: $N \leftarrow$ GetNeighbors $(x) / /$ find number of neighbors of node $x$

2 : weight $\leftarrow$ GetWeight $(x) / /$ find weight of node $x$ and all other nodes

3: If $|\mathrm{N}| \geqslant \min$ Node

4: Select $x$ as a core point

5: if weight $(x) \leq$ weight $(y), \forall y \in N$

6: $\quad$ select $x$ as $\mathrm{CH}$

7: $\quad$ minNode $=|N|$

8: end if

9: else

10: select $x$ as border point

11: end if

12: for each $y \in N$ do

13: if $y$ is not $\mathrm{CH}$ nor member of any cluster

14: $\quad$ if $\left(\mathrm{MPC}_{x, y} \geq \mathrm{MPC}_{\mathrm{g}}\right)$

15: $\quad C \leftarrow\{y\}$; include $y$ in the cluster $C$

$\operatorname{ODCR}\left(y, M P C_{g}\right.$, minNode $)$

else

19: end if

20: end if

21 return $\mathrm{CH}$

Protocol for emergency sensor applications

In Algorithm I, each node $x$ calculates their weight and number of neighboring nodes and broadcasts to its neighboring nodes (line 1-2). Then, the node $x$ whose weight is the lowest among all neighboring nodes becomes cluster head $(\mathrm{CH})$ of cluster $\mathrm{C}$ (line $5-6)$. All other nodes $y$ who are neighboring nodes of $x$ but is not selected as $\mathrm{CH}$ or member of any cluster will compare $M P C_{x, y}$ with global $M P C_{g}$. If the $M P C_{x, y}$ is greater than or equal to $M P C_{g}$ node $y$ is included as a member node of cluster $C$ (line 12-15) and the same process continues (line 16).

\subsection{Steady phase (data routing)}

In this protocol, once clusters are formed and isolated nodes (i.e., noise) are identified data transmission or routing starts.

Whenever a node $x$ receives a data packet, (1) if it is a member of a cluster it communicates with the $\mathrm{CH}$, transmits data to the $\mathrm{CH}$. Then the $\mathrm{CH}$ transmits data to the next hop towards the destination. (2) if the node $x$ is a border node, it is a noise, which can either be a member of a cluster or not. If $x$ is a member of the cluster the above rule 1 applies. If the node $x$ is not a member of a cluster it must be within the communication range of two neighboring clusters. Then, the node $x$ broadcasts a message to find out the closest neighboring cluster and a forwarder node that is closest to the destination to transmit data. Then the node $x$ joins to a cluster by restoring connectivity (presented in Section 4.3). The node might also be lost contact with other nodes and become isolated. Figures $2-4$ illustrate 
this scenario. In Figure 2, the node $A$ is not a member of cluster. Whenever it senses or receives data forwards to the closest forwarder node $B$. However, the node $B$ has not contact with other nodes. As it moves and reaches within the communication range of node $C$, it immediately forwards data to node $C$ (Figure 3 ). Then, node $\mathrm{C}$, being a noise (border node trying to join to a cluster), forwards data to the member nodes and $\mathrm{CH}$ of a cluster towards the destination as illustrated in Figure 4.

Each node keeps the MPC value with all of its neighboring nodes and opportunistically forwards data to the neighboring node with the highest MPC. As this protocol is applicable to disaster situation where all nodes are moving almost at the same speed towards the location of emergency or disaster they will more likely to keep communicating a number of nodes. Thus, the source node should be opportunistically able to transmit data to the destination by communicating with those nodes.

\subsection{Cluster restoration}

As nodes are most likely mobile in opportunistic networks, they reach at the border of the cluster or become isolated. Hence, cluster restoration is required. We present two scenarios below where cluster restoration is performed.

Scenario 1: once the node $x$ becomes border node of cluster $C$ it must runs an algorithm of cluster restoration or join other cluster that maintains the global $M P C$ increasing the communication range $R c$ of $y$ as follows.

$$
M P C_{y, x}=M P C_{g}=\frac{R_{c}-d}{V_{x}+V_{y}}
$$

where $V_{y}$ and $V_{x}$ are the velocity of the border nodes $x$ and $y$ of two neighboring clusters and $\mathrm{Rc}$ is the communication range of nodes in both clusters. Figure 5 demonstrates scenario 1.

Scenario 2: If $x$ becomes isolated the algorithm finds the minimum MPC between $\mathrm{MPC} x, y$ and $\mathrm{MPC} x, C$ and increases $R c$ of this minimum to maintain the global MPC.

The cluster that will participate in cluster restoration depends on the weight of its $\mathrm{CH}$ calculated using Eq. (5). If the weight of the $\mathrm{CH}$ of the cluster is the lowest it will activate its border node for instance, $y$ in this above scenario to tract the connectivity with the cluster $C$, as illustrated in Figure 6. Algorithm II presents the pseudocode of cluster restoration based on scenarios 1 and 2 .

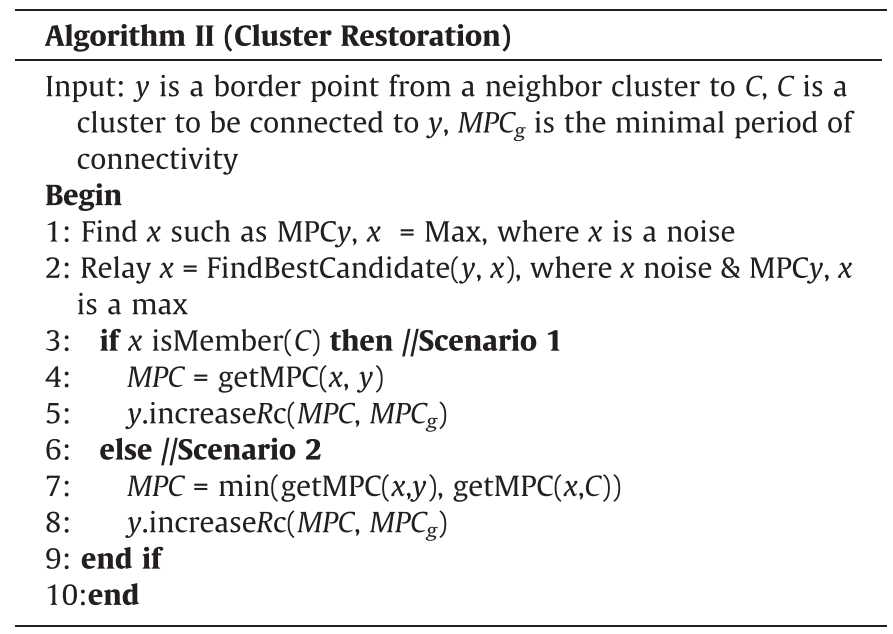




\section{Performance evaluation}

This section presents the performance evaluation of the proposed ODCR protocol.

\subsection{Computation efficiency}

The computational and message complexity of the proposed ODCR protocol is O(1) per node. Initially, each node broadcast one message of its velocity and its position to neighbors. Then, once $\mathrm{CHs}$ are selected each $\mathrm{CH}$ broadcasts one declaration message (such as "hello message" as presented above). When the $k$-hop nodes $(k \geq 1)$ hear the declaration message they broadcast an acknowledgement message and join to the cluster and rebroadcast the declaration message to the $k+1$-hop nodes to find the shorted path to the destination. Hence, it has been observed that each node sends at most three messages to perform the operation.

\subsection{Energy efficiency}

To identify the energy efficiency of the proposed ODCR protocol we analyze and compare the ODCR protocol with some benchmark clustering protocols such as LEACH [18] and TEEN [19] protocols and also a very recent opportunistic routing protocol, called LORA [21].

The cluster formation phase is initiated in LEACH and TEEN protocols after a certain number of rounds where all nodes participate in the cluster restoration or reformation. On the other hand, cluster restoration is initiated in the proposed ODCR protocol only when a node becomes a border node or becomes isolated. Also, only the border nodes and two neighboring $\mathrm{CHs}$ participated in this cluster restoration process. Other nodes remain in their cluster and do not participated in the cluster formation process.

Hence, the proposed ODCR protocol seems to be more energy efficient, lower packet loss ratio and higher throughput as compared to the existing LEACH and TEEN protocols. Let us assume that

The number of nodes in the network $=n$

The number of clusters in the network $=c$

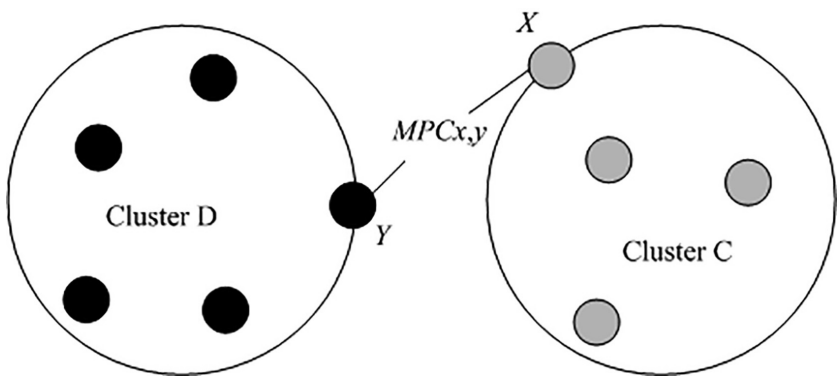

Figure 5.

Scenario 1 , where $x$ is a

border node of cluster $\mathrm{C}$.

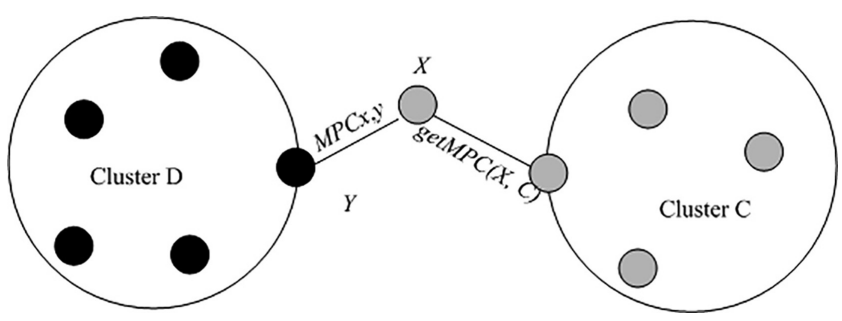

Figure 6.

Scenario 2, where $x$ is not a member of $\mathrm{C}$. 
Then, the average number of nodes in each cluster $=\frac{n}{c}$

The number of messages each node transmits and receives for cluster formation $=m$

The average number of message transmission for cluster

$$
\text { formation }=n \times m
$$

\section{For cluster restoration in ODCR protocol}

The number of nodes participate in cluster restoration $=3$ where $3<n$

The average number of message transmissions in ODCR for

$$
\text { cluster restoration }=3 \times m
$$

Hence, the number of transmitted messages for cluster restoration is of $\mathrm{O}(n)$.

Comparing equations (6) and (7), we expect that the average number of message transmissions in ODCR protocol is much lower than that in LEACH and TEEN protocols.

On the other hand, LORA [21] is a non-clustered multi-hop opportunistic routing protocol.

Let us assume that the number of hops from source to destination in LORA $=h$

$$
f_{1}+f_{2}+f_{3}+\ldots \ldots \ldots+f_{h-1} \leq n
$$

where, $f_{i}$ represents the number of forwarder nodes at hop $i$.

The total number of message transmissions for selecting forwarder nodes will be about

$$
f_{1} \times m_{1}+f_{2} \times m_{2}+f_{3} \times m_{3}+\ldots+f_{h} \times m_{h}
$$

where, $m_{i}$ represents the number of message transmission for selecting forwarder nodes at hop $i$.

If $b_{1}, b_{2} \ldots \ldots, b_{h}$ represent the number of message transmission for selecting the best forwarder node at hops $1,2 \ldots \ldots, h$, respectively the total number of message transmission would be

$$
f_{1} \times\left(m_{1}+b_{1}\right)+f_{2} \times\left(m_{2}+b_{2}\right)+f_{3} \times\left(m_{3}+b_{3}\right)+\ldots+f_{h} \times\left(m_{h}+b_{h}\right)
$$

Hence, selection of forwarder nodes for transmitting a data packet from the source to destination will be of $O\left(n^{2}\right)$. The process of selecting such path with forwarder nodes occur every time a node transmits data to the destination. Hence, it is more likely that the total number of message transmission in LORA protocol is much more than that in the proposed ODCR protocol. We will perform an experiment (simulation) to verify this.

As energy consumption of sensor nodes is directly proportional to the number of transmitted messages the energy consumption of ODCR protocol is expected to be much lower than that in the LEACH, TEEN and LORA protocols. Similarly, as the number of message transmissions is much more in LEACH, TEEN and LORA protocols the probability of packet loss in these protocols will be higher than that in the proposed ODCR protocol.

\section{Simulation setup and results}

We perform a simulation to evaluate the performance of the proposed opportunistics routing protocol and compare with some existing well known routing protocols. We use the network simulator NS-3 [12] to perform the simulation. The following sections present the energy, network and mobility models along with the simulation results. 


\subsection{Energy model}

Energy consumptions to transmit a packet of size $n$ over distance $d$ is given by

$$
E_{T X}=n * \varepsilon_{\text {elec }}+n * \varepsilon_{f s} * d^{\alpha}
$$

Energy required receiving a packet is

$$
E_{R X}=n * \varepsilon_{\text {elec }}
$$

where, $\varepsilon_{\text {elec }}$ and $\varepsilon_{f s}$ in Eqs. (11) and (12) represent energy spent in transmitter electronics circuitry and energy spent in RF amplifiers for propagation loss. The constant (propagation loss exponent) $\alpha$ is dependent on the surrounding environment. For free space without any obstruction in the line of sight, $\alpha=2$.

\subsection{Network and mobility model}

The sensor network comprising $N$ nodes is represented by a graph $G(V, E)$ where $V$ represents a set of sensor nodes (vertices) and $E$ represents a set of communication links (edges). Two nodes, $u$ and $v$, communicate with each other if they are within their communication range $(R c)$. This property is known as a Unit Disk Graph (UDG) of graph theory. However, since our work is proposed in the context of sensor-based opportunistic networks, we consider the "double range property" [24] in our experiment defined as $R c=n \times$ $R s$ where $n \geq 2$ and $R s$ refers to the sensing range.

We consider $4.0 \mathrm{~km}^{2}$ (approximately $2 \mathrm{~km} \times 2 \mathrm{~km}$ ) network size as it is a realistic area around the disaster zones where rescue personnel need to be connected for the recovery operation. Moreover, the mobility speed of rescue personnel is assumed to be about 5 meter/ second or 300 meter/minute considering the scenario that rescue personnel are moving slow or walking in a certain region of that area to perform the operation.

The random mobility of the nodes was generated using mobility generator BonnMotion [25], which places each node randomly in a position in the simulation area of size $2000 \times 2000$ meter ${ }^{2}$. The pattern of mobility is the Random Scenario (RS) provided by the mobility scenario generator BonnMotion [25]. The generated mobility patterns pauses each node at its current location for a certain period before moves to another randomly generated location at random speed. As sensors are used in simulation, we consider the IEEE 802.15.4 Zigbee protocol in the network model. Table 1 lists simulation parameters and their respective values.

\subsection{Performaces metrics}

The performance metrics that are used to measure the performance of the proposed ODCR protocol are defined as follows.

\begin{tabular}{ll}
\hline Parameter & Value \\
\hline Simulation network area & 2000 meter $\times 2000$ meter \\
Number of node & 100 \\
Communication range of a node & 200 meter \\
Transport protocol & UDP \\
MAC protocol & IEEE 802.11 \\
Mobility Model & Random \\
Mobility Speed & 5 meter/second \\
Pause time & $1 / 2$ second \\
Duration of a round & 200 seconds \\
Traffic type & Constant Bit Rate (CBR) \\
Data transmission rate & 250 Kbit/second \\
Data packet size & 128 Bytes $=1$ Kbit
\end{tabular}

Protocol for emergency sensor applications

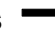


Energy Consumption - is the total energy consumed by the sensor nodes in the network over a number of rounds.

Throughput - is the amount of data transmitted by a node per second. We calculate throughput in Gigabytes/second in this paper.

Packet Delivery Ratio - is the ratio of the total number of packets delivered to the total number of packet transmitted.

Packet Loss Ratio - is the ratio of the total number of packets lost to the total number of packets transmitted.

\subsection{Experimental results}

Figures 7-10 illustrate the performance of the proposed ODCR opportunistic routing protocol in terms of network energy consumption, packet loss, throughput, and packet delivery ratio. Each simulation runs for $200 \mathrm{~s}$. We evaluate the performance of the proposed ODCR protocol over a number of rounds. The output is the average of output received a certain number of rounds.

Figure 7 demonstrates that the energy consumption of the proposed ODCR protocol is much lower than the existing TEEN and LEACH clustering protocols. This is because using Eqs. (6) and (7) we find that the average message transmission in ODCR is much lower than that in LEACH and TEEN protocols. Similarly, the number of message transmissions in ODCR protocol is also lower than LORA protocol (as analyzed using Eq. (10)). Hence, the network energy consumption in the proposed ODCR protocol is lower than that in LORA protocol.

Figure 8 demonstrates that the packet loss ratio of the proposed ODCR protocol is much lower than the existing LEACH [18], TEEN [19] and LORA [21] protocols because the proposed ODCR protocol can perform quick cluster recovery due to node movements as compared to the existing algorithms. Hence, the probability of packet loss is much lower in ODCR approach that results in lower packet loss. More specifically, in TEEN protocol, clusters are reconstructed only at the end of each round regardless of any node moves out of

Figure 7.

Comparison of Energy Consumption.

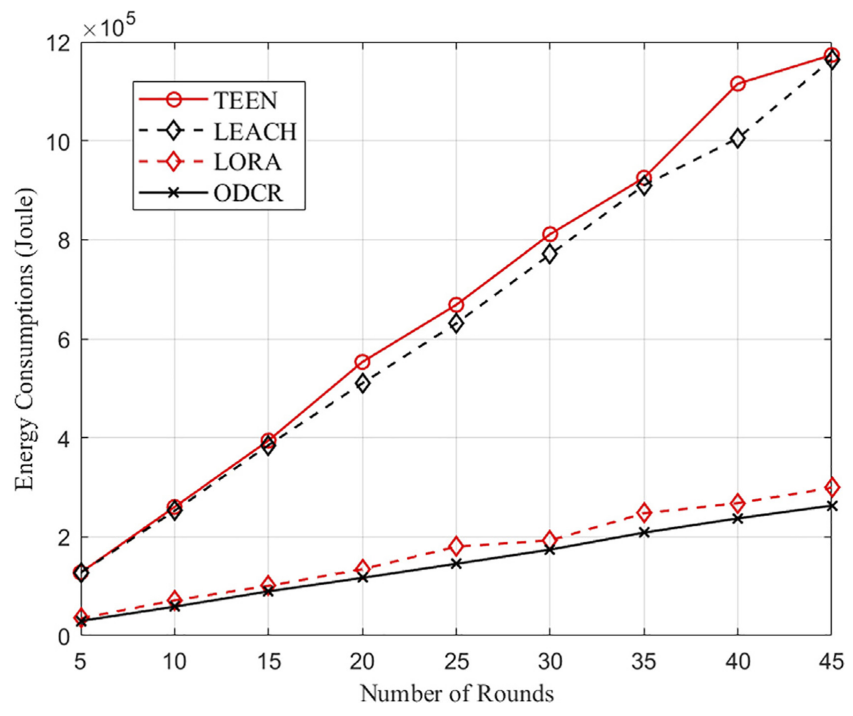




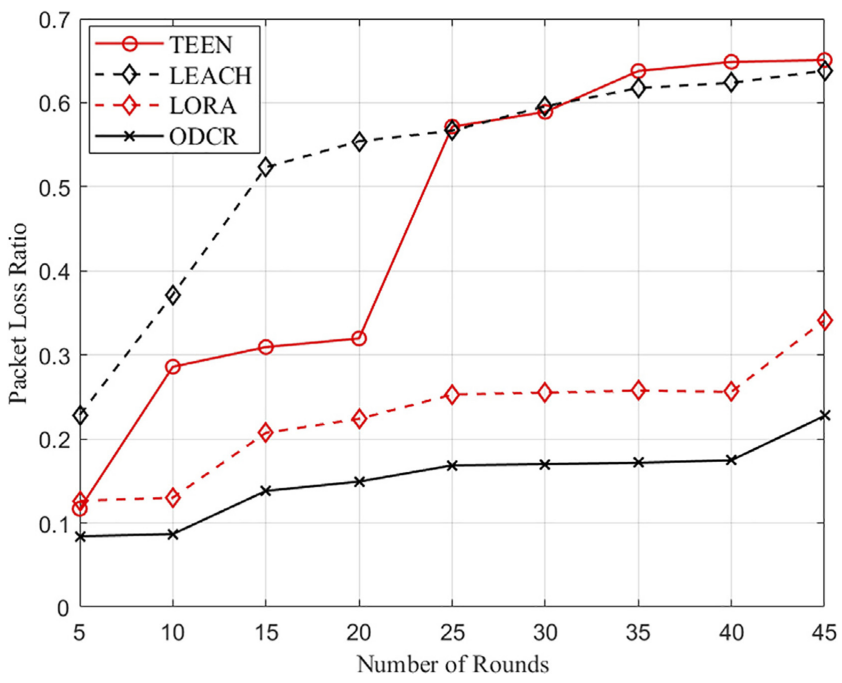

Protocol for emergency sensor applications

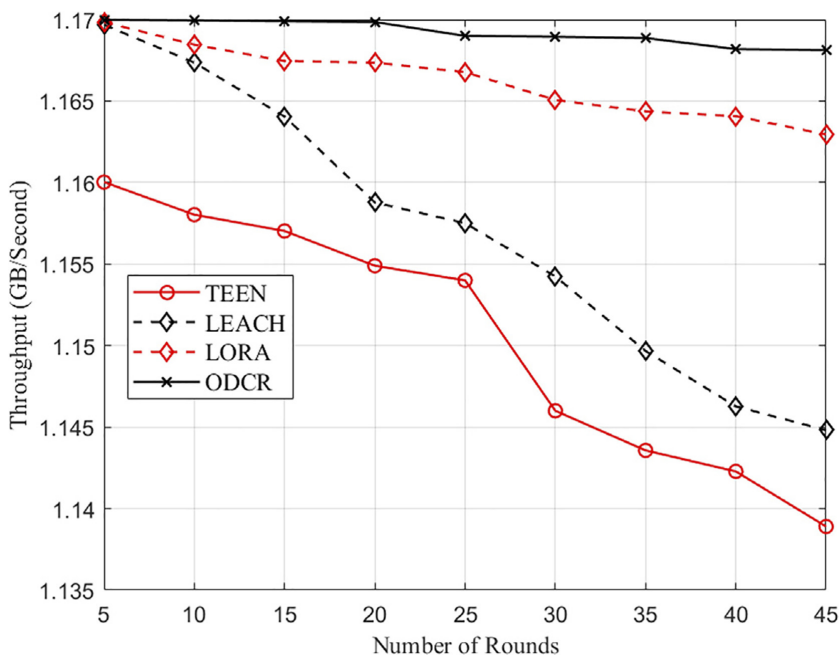

Figure 8. Comparison of Packet Loss Ratio.

Figure 9. Comparison of Throughput.

the cluster in a round. In LEACH, clusters are reconstructed at the end of a certain number of rounds. Even if a number of nodes move out of clusters and become isolated or noise the clusters are not formed unless the number of rounds reach. Hence, the probability of packet loss is much higher in LEACH and TEEN protocols. This also results in lower throughput in LEACH and TEEN protocols. On the other hand, in LORA protocol, a candidate zone is constructed with a set of forwarder nodes for each hop. Moreover, the best forwarder is selected for each candidate zone to create a path from source to the destination for transmitting data packets. As the nodes are mobile the probability of moving the forwarder nodes in this preselected path is high. Hence, the probability of packet loss is also high in LORA protocol. 


\section{$\mathrm{ACI}$}

Figure 10.

Comparison of Packet Delivery Ratio.

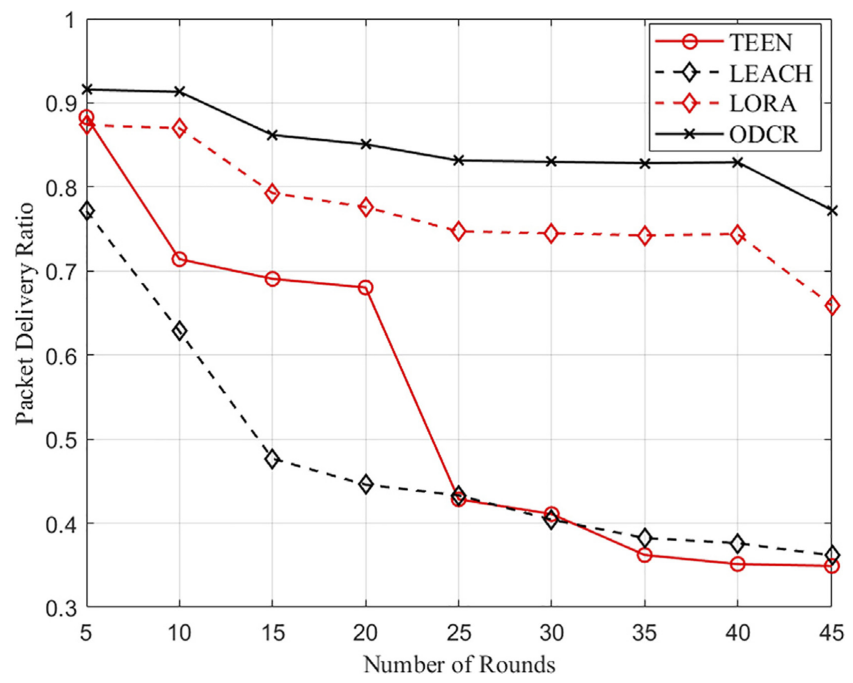

Figure 9 demonstrates that the throughput of the proposed ODCR protocol is much higher than that in LEACH, TEEN and LORA protocols. This is because the proposed ODCR protocol performs cluster recovery and creates clusters rapidly based on the worst-case scenario of node movements between two adjacent nodes. Existing approaches loss connectivity in such scenario, which is not the case for proposed ODCR protocol.

Similarly, Figure 10 illustrates that the ODCR protocol achieves higher packet delivery ratio as compared to LEACH, TEEN and LORA protocols because the proposed ODCR protocol maintains connectivity in most of the mobility scenarios. In the simulation results, we find that on that average packet delivery ratio is almost $50 \%$ more in ODCR protocol as compared to LEACH and TEEN protocols and about 15\% more in ODCR protocol as compared to LORA protocol.

Moreover, the total amount of data transmitted is also proportional to the total number of message transmitted with the assumption that the size of a data packet is fixed. If the total amount data transmitted in LEACH, TEEN and LORA protocols is much more than that in the proposed ODCR protocol the total time required to transmit that amount of data (i.e., latency) will also be more in LEACH, TEEN, LORA protocols than in ODCR protocol. We will perform an experiment to verify this claim as a part of our future research in this area.

\section{Conclusion and Future work}

This paper presents ODCR - an energy efficient and reliable opportunistic, density-clustered based routing protocol. This protocol opportunistically transmits data packets among sensors and other wireless devices in disaster and emergency situations. In this ODCR protocol, the concept of density-based clustering is used to create a number of clusters comprising sensors or wireless devices. Each sensor or wireless device joins to a cluster and transmits data packets to the cluster head or if the node is isolated (i.e., not a member of a cluster) opportunistically forwards data packets whenever encounters another node with higher probability of data delivery to the destination. We evaluate the performance of the proposed ODCR routing protocol in terms of network energy consumption, packet loss, 
throughput and packet delivery ratio and compare with existing well-known LEACH, TEEN and LORA protocols. Experimental results find that the performance of the proposed ODCR routing protocol is much better than that in LEACH, TEEN and LORA protocols in terms of those performance metrics. However, we plan to verify the performance of the ODCR protocol in terms of latency, localization error and other performance metrics in future.
Protocol for emergency sensor applications

\section{References}

[1] Hung-Chin Jang, Yao-Nan Lien, Tzu-Chieh Tsai, Rescue information system for earthquake disasters based on MANET emergency communication platform, IWCMC, 2009.

[2] Maneesha V. Ramesh, Anoop Jacob, Aryadevi R. Devidas, Enhanced emergency communication using mobile sensing and MANET, ICACCI, 2012.

[3] C. Zheng, L. Chen, D. Sicker, Hybrid cellular-MANETs: An energy-aware routing design, in: Wireless On-demand Network Systems and Services (WONS), 2014 11th Annual Conference on, Obergurgl, 2014, pp. 9-16.

[4] H. Verma, N. Chauhan, MANET based emergency communication system for natural disasters, in: Computing, Communication \& Automation (ICCCA), 2015 International Conference on, Noida, 2015, pp. 480-485.

[5] Z. Lu, X. Sun, T.L. Porta, Cooperative data offloading in opportunistic mobile networks, in: IEEE INFOCOM 2016 - The 35th Annual IEEE International Conference on Computer Communications, San Francisco, CA, 2016, p. 19.

[6] Z. Lu, G. Cao, T.L. Porta, Networking smartphones for disaster recovery, in: 2016 IEEE International Conference on Pervasive Computing and Communications (PerCom), Sydney, NSW, 2016, pp. 1-9.

[7] M. Ester, H. Kriegel, J. Sander, X. Xu, "A density-based algorithm for discovering clusters in large spatial databases with noise", in: Proceedings of the 2nd International Conference on Knowledge Discovery and Data mining, 1996, pp. 226-231.

[8] Ramon Sanchez-Iborra, Maria-Dolores Cano, JOKER: a novel opportunistic routing protocol, IEEE J. Selected Areas Commun. 34 (2016) 1690-1703, https://doi.org/10.1109/JSAC.2016.2545439.

[9] M. Ester, H. Kriegel, J. Sander, X. Xu, A density-based algorithm for discovering clusters in large spatial databases with noise, in: Proceedings of the 2nd International Conference on Knowledge Discovery and Data mining, AAAI Press, 1996, pp. 226-231.

[10] I.F. Senturk, K. Akkaya, Connectivity restoration in disjoint Wireless Sensor Networks using centrality measures, in: 39th Annual IEEE Conference on Local Computer Networks Workshops, Edmonton, AB, 2014, pp. 616-622.

[11] J.E. Said, L. Karim, J. Almhana, A. Anpalagan, Heterogeneous mobility and connectivity-based clustering protocol for wireless sensor networks, in: Intl conf. on communications (ICC), Sydney, 2014, pp. 257-262.

[12] Payal Jadhav, Rachna Satao, A survey on opportunistic routing protocols for wireless sensor networks, Procedia Computer Science 79 (2016) 603-609, https://doi.org/10.1016/Zj.procs. 201603076, 1877-0509.

[13] M.W. Kang, Y.W. Chung, An energy-efficient opportunistic routing protocol in delay tolerant networks, in: 2016 International Conference on Information and Communication Technology Convergence (ICTC), Jeju, 2016, pp. 655-659.

[14] R.M. Santos, J. Orozco, S.F. Ochoa, R. Meseguer, D. Mosse, Providing real-time message delivery on opportunistic networks, IEEE Access 6 (2018) 40696-40712, https://doi.org/10.1109/ACCESS 2018.2848546.

[15] M.W. Kang, Y. Kim, Y.W. Chung, An opportunistic forwarding scheme for ICN in disaster situations, in: 2017 International Conference on Information and Communication Technology Convergence (ICTC), Jeju, 2017, pp. 948-950, https://doi.org/10.1109/ICTC.2017.8190821. 
[16] Arati Manjeshwar, Dharma Agrawal, TEEN: ARouting protocol for enhanced efficiency in wireless sensor networks, in: Intl. Proc. of 15th Parallel and Distributed Processing Symp., 2001, p. 189, https://doi.org/10.1109/ IPDPS.2001.925197.

[17] M. Bani Yassein, Y. Khamayseh, W. Mardini, Improvement on LEACH protocol of wireless sensor network (VLEACH), Int. J. Digit. Content Technol. Appl. (2009) 132-136.

[18] W. Heinzelman, A. Chandrakasan, H. Balakrishnan, Energy-efficient communication protocol for wireless microsensor networks, Proceedings of the 33rd Hawaii International Conference on System Sciences, 2000.

[19] A. Manjeshwar, D.P. Agrawal, TEEN: a routing protocol for enhanced efficiency in wireless sensor networks, Proceedings of the $15^{\text {th }}$ International and Distributed Processing Symposium, 2001.

[20] Y. Han, H. Wu, Z. Yang, D. Li, A new data transmission strategy in mobile D2D networksDeterministic, greedy, or planned opportunistic routing?, IEEE Trans Veh. Technol. 66 (1) (2017) 594-609, https://doi.org/10.1109/ TVT.2016.2540641.

[21] A. Hawbani, X. Wang, Y. Sharabi, A. Ghannami, H. Kuhlani, S. Karmoshi, LORA: load-balanced opportunistic routing for asynchronous duty-cycled WSN, IEEE Trans. Mobile Comput. 18 (7) (2019) 1601-1615, https://doi.org/10.1109/ TMC.775510.1109/TMC.2018.2865485.

[22] B.K. Saha, S. Misra, S. Pal, SeeR: simulated annealing-based routing in opportunistic mobile networks, IEEE Trans. Mobile Comput. 16 (10) (2017) 2876-2888, https://doi.org/10.1109/TMC. 2017.2673842.

[23] J. So, H. Byun, Load-balanced opportunistic routing for duty-cycled wireless sensor networks, IEEE Trans. on Mobile Comput. 16 (7) (2017) 1940-1955, https://doi.org/10.1109/TMC.2016. 2606427.

[24] G. Xing, C. Lu, R. Pless, Q. Huang, Impact of sensing coverage on greedy geographic routing algorithms, IEEE Trans. Parallel Distributed Syst. 17 (4) (April 2006) 348-360.

[25] N. Aschenbruck, R. Ernst, E. Gerhards-Padilla, M. Schwamborn, BonnMotion: a mobility scenario generation and analysis tool Article 51, in: Proceedings of the 3rd International ICST Conference on Simulation Tools and Techniques (SIMUTools '10). ICST (Institute for Computer Sciences, Social-Informatics and Telecommunications Engineering), Brussels, BEL, 2010, pp. 1-10, https:// doi. org/10.4108/ICST.SIMUTOOLS2010.8684.

\section{Corresponding author}

Lutful Karim can be contacted at: lutful.karim@senecacollege.ca

For instructions on how to order reprints of this article, please visit our website:

www.emeraldgrouppublishing.com/licensing/reprints.htm

Or contact us for further details: permissions@emeraldinsight.com 